\title{
The Impact of Role Models on Self-identity of LGBTQ Teenagers
}

\author{
Yutong Liu ${ }^{1, \text { a, }}{ }^{*}, \dagger$, Zhijing Jiang ${ }^{2, \text { b, }{ }^{*}, \dagger}$, Hanqing She ${ }^{3, c, ~}{ }^{*} \dagger$, Youan Zhang ${ }^{4,}$ d, *, \\ ${ }^{1}$ Shanghai United International School, Shanghai, 201100, China \\ ${ }^{2}$ College of International Education, Shenzhen, 518043, China \\ ${ }^{3}$ Jinling high school Hexi campus, Nanjing, 210000, China \\ ${ }^{4}$ Shanghai Weiyu International High School, Shanghai, 200438, China \\ *Corresponding author email: ${ }^{a}$ s16245.Jiang@stu.scie.com.cn; ${ }^{b}$ amb0528@foxmail.com; ${ }^{2} 2905300235 @ q q . c o m$; \\ damyzhangya@qq.com \\ These authors contributed equally.
}

\begin{abstract}
Combing the theory of role models (a figure other people look up to in order to help determine appropriate behaviors) and the theory of teens identity, an interesting correlation about the effect of social media is found to both of them. This paper have the speculation that role models help identity in teens. This paper focuses on the group of LGBTQ teenagers. The use of questionnaires and interviews helps to find out that the development of social media. Specifically, with the increase in LGBTQ role models, more teenagers are able to identify themselves about their own sexuality. Based on the data, it proves that most of the LGBTQ teenagers believe role models help them in a positive way. According to the evidence, the new generation is much opener and firmer in identifying themselves. Hence, this paper estimates that the improvement in teens self identity cannot leave the help of role models in social media. It is expected that the development of social media, more teenagers can be able to distinguish themselves and be accepted.
\end{abstract}

Keywords: role models, LGBTQ, self-identity

\section{INTRODUCTION}

In the society which encountered countless bombardment of information nowadays, medias have hugely dominated the ideology of human beings. Generation Z (1995-now) has lived in a media world since they born. Their everyday life especially the high school teenagers nowadays (by this time refers to high school students born around 2005) is affected by those active influencers online who are also known as $\mathrm{KOL}$ (Key opinion leaders), which is defined as "a person or organization who has expert product knowledge and influence in a respective field". Moreover, since more and more rising online users are joining several platforms, KOLs from a wide assortment of cultures incorporates into unity. Hence, it leads to a more tolerable society, including the embracement of color, women empowerment and more diversified sexualities. The group focuses on the topic of LGBTQ+, examining the impacts of LGBTQ+ KOLs/role models to LGBTQ+ teenagers by the means of social media. We hypothesized that those LGBTQ+ teenagers 'self identity could be altered partially due to the role models. Additionally, with the presence of those role models, their self recognition can be effected by themselves personally and the surrounding since those two aspects' counterparts in identity recognition refers to individual identity and collective identity respectively.

\section{HISTORY AND SELF-IDENTITY OF LGBTQ TEENAGERS}

Years over years, self-identity of teenagers is always a problem throughout history. Self-identity is the recognition of one's potential and qualities as an individual, especially in relation to social context. Teenagers, no matter what countries they belonged to or what background they had, were always seeking for status symbols (an expression of self-identity), which included clothing and possessions to create a sense of positive affiliation. These actions appeared as a certain behaviors that some teens believe that appearing mature will bring acceptance, involving smoking, drinking, drugs, and sexual activities.[8] These activities established the later 
formation of different subcultures and minority interests. Moreover, the behaviors of rebellion, the presence of idols, and the formation of cliques also help to express their way of identifying themselves. Overall, a teen's identity is the result of various internal and external factors. Though a teen has some control over their identity development, teen identities are also formed by environmental forces outside of their control: peers, family, school, ethnic identity, and other social environments. Self-identity develops as teens try out different roles and attitudes in different settings, e.g., home, school, and social atmospheres, which allows teens the opportunity to explore their own values, belief systems, personal ethics, spirituality, sexuality, and gender.[7] Among these factors, role models are especially affective. Some teens may identify with a famous person, e.g., try to become like that person. According to a developmental psychologist, James Marcia, teen identity development occurs in response to crises in domains such as school, relationships, and values. Especially at the age from 13 to 19 , teenagers have just passed through the time with the conflict between identity and role confusion. These are the years for teenagers to find their own identity, and not until 20 years old, they are able to establish intimacy isolation. The age groups effectively help define the years that a person can be mostly affected in deciding its own identity. Besides these teen identities among "normal" teenagers, for teenager minority groups, the progress of receiving identity becomes much more difficult. The group of LGBTQ teenager is a typical example.[9][10]

LGBTQ is a term for lesbian, gay, bisexual, transgender and queer or questioning. [8] These terms are used to describe a group of person's sexual orientation or gender identity. The identity of this group is shown by the existence LGBTQ Pride Month. LGBTQ Pride Month is commemorated each year in the month of June, as a large event of every LGBTQ standing up and expressing their "pride" of becoming one of the rare sexualities. This is a form of identifying themselves through presenting their existence to the whole society and to the ones that oppose the existence of LGBTQ. As this group is always lack of acceptance from the world, just like any other minorities, it is hard for some young members to identify themselves as one of them. This makes the existence of role models, like educators, civic leaders, mothers, fathers, clergy, peers, and ordinary people encountered in everyday life, extremely vital. Especially for role models from the internet, they accurately figure out the taste of young teens (LGBTQ teenagers), and help them identify themselves. This cannot leave the help of data collection of social medial. However, since teen identity is already a hard major process for teenagers, the identity of being one of the minorities will be confused and puzzled, not only for LGBTQ teenagers, but also for the ones who are still uncertain for their sexuality. It happens to be on many popular softwares, like Utube, Bilibili, Tiktok etc. Idols or the role models of minority group helps the identity of different people. More and more teenagers are able to keep in touch with such existence, and they seem to be more familiar with their status symbols than the last generations. The increase number in "coming out" teenagers years by years have raised the social value about LGBTQ. Some experts believe it is the development of human beings about cultural pluralism, while others believe this is due to the popularization of internet in the form of social media.

These is evidence showing that the existence of internet, which leads to increasing number in role models, does help to extend the barrier of the traditional mode of communication and provides a much larger platform and way which access to more information.[7] As young teenagers love to contact with new things on the internet, the youth subculture and minorities, such as LGBTQ group, gradually increases into more daily life than before. It happens to be more minorities have become existed in real life. Since years before, the minorities are not brave enough to from their own identity group in the society as they don't know how many same-kind they have, the development of social media helps broaden the size of such group and more members are willing to speak out. This is one of the speculations about the recent increase in numbers of "coming out" LGBTQ teenagers. The phenomenon then raises wonder about the affect of role models from internet to the self-identity of LGBTQ teenagers.

\section{RESEARCH DETAILS AND NOWADAYS TENDENCY}

One ubiquitous phenomenon observed today is that comparing to heterosexual youngsters, LGBTQ+ teenagers are more active online especially on social media. This makes us feel curious about the relationship between social media and LGBTQ+ teenagers. We focus on the LGBTQ+ role models on social media, studying their impact on LGBTQ+ ordinary teenagers' selfidentity recognition. The Execution divided in two parts an LGBTQ+ interview regarding to common conceptual questions, and a questionnaire that includes our final intentions.

According to JiaJun Tao's essay regarding to identity introductory theory, he divided identity recognition into two parts: self-identity and social identity, which includes individual identity and collective identity. Selfidentity emphasizes individual as the essential recognition factor, while social identity concerns one individual's social nature more.

In the interview and questionnaire, we settled questions from these two aspects, i.e., there are some more personal questions pointing directly to the interviewee themselves, leading to a trend in individual 
identity. Moreover, there are also questions relating to a wider range, to be specific, the surrounding like role models and social media. These questions contribute to understandings about collective identity. Finally, the overall analysis would be generated by synthesizing the data answers in two identity aspect.

\section{INTERVIEW INTRO}

The LGBTQ+ interview has contained 50 samples; all of the interviewees are high school students. We used convenience sampling through Wechat. Most of them have realized their sexuality in secondary school. A few of them have realized even earlier. The group compiled 10 leading questions about LGBTQ+ role models. The goal for interview is to get familiar with LGBTQ+ teenagers' ideologies and receive some information toward the LGBTQ+. We settled questions regarding to LGBTQ+ role models, and can concluded by saying that most of the interviewees have been aware of those influencers online. Most homosexual students treat them as quite important role, as those brave people are willing to stand out and supporting uncommon sexualities. Those online models made positive impacts on them by giving them power, popularizing relevant knowledge and strengthening teenagers' identity recognition thereby resulting in their greater confidence in the real life as being a member of LGBTQ+. Nevertheless, it is also those famous people's large influence that may produces some consequences especially stereotypes and identity authenticity.

\section{【LGBTQ+ INTERVIEW RESULTS】}

According to a high school lesbian interviewee, she initially starts to know LGBTQ+ when her friend comes out to her. From that moment, she began to comprehend about a totally different concept, i.e., a culture with more diversified sexualities. Due to the big data, algorithmic recommendations started to make suggesting articles about LGBTQ+, including many encouraging films posted by LGBTQ+ couples. By witnessing those LGBTQ+ Youtubers and bloggers's videos and the comments below, she thinks the young public mostly accept the culture though she might not see the whole. Meanwhile, she recalled a girl who has received her appreciation during secondary school. The interviewee has finally realized that the feeling she had for this girl was not simply admiration, but affection. From that moment on, she became somewhat clearer with her identity as she had eventually figured out her sexuality, which mostly result from the impact of LGBTQ+ role models on social media. The group has also asked interviewees regarding to the influence of role models on their sexualities.

To begin with, more than $90 \%$ interviewees have mentioned enhancing identity recognition and popularizing relevant knowledge as the two major benefits resulting from the online celebrities. To be specific, one interviewee stated that she feels more comfortable with her identity when she saw many people same as her are sharing their life online. Many people are living happily and proud of being a member of LGBTQ+. This makes her more confident in real life since she feels like getting support. Furthermore, because there are a diversified kind of people online, other interviewees have a greater possibility of exploring the right person. In detail, they can find the kind of person they like whether the overlook or intrinsic traits, through the great media.

Furthermore, role models can use their influence to spread more information about LGBTQ+. Since all interviewees are high school students, a majority of them are being shy to ask or come out of the closet in real life. When many queries such as sex life or history of LGBTQ+ pop up through those teenager' mind, learning online is the optimal way in order to ensure the privacy. One of the interviewees has said that she learned the fluid sexuality online by subscribing a renowned lesbian Youtuber.

However, the rise of popularity in media can also lead to several consequences, including but not limiting to stereotype and suspect on the authenticity of user's real identity. For one thing, quite a lot of people casts doubt on the presence of LGBTQ+, they don't believe people with same sex can be loved. For instance, stereotypes may include "gay is girly", "homosexuals don't treat love seriously", "the impossibility to distinguish friendship and love within same sex". For another, since LGBTQ+ has received more and more attentions online nowadays, a phenomenon exists: some bloggers tend to gain more interests by pretending themselves as members of LGBTQ+ to be "special". Though the real members can enlarge the influence and making the community bond closer together, it makes audience feel a bit more puzzling about the identity of numerous so-called "homosexual" virtual images online.

\section{Questionnaire intro}

After we have gathered the results from interview, we made an anonymous questionnaire for LGBTQ+ teenagers in order to test the universality of the phenomenon gathered from previous interview. We altered some of the questions and focus on asking about role models' influence on LGBTQ+ teenagers. The question type becomes more diversified once we made it into a questionnaire: including scale rating, agree or disagree (attitudes towards the phenomenon) and some free responses. The questionnaire collected 70 samples. When the data are collected, we synthesize them and draw to the conclusion that most of the common statements in the previous interview have been proved. Around $70 \%$ of people confessed that LGBTQ+ role models play a relatively huge role in their life while others don't. Among those who did, when asking about the impact of social 
media, more voices in claiming the positive impacts are present than the negative impacts.

Role models on the internet are influential figures in helping the LGBTQ community in various ways. For instance, those role models could help people to overcome their confusion of their sexual orientation, they could popularize knowledge that are relate to sexual minority groups using their influential power on the internet, they could also play an crucial role in translating or communicating information between LGBTQ community and the majority others. Real-life examples could most effectively support the claim of role models being positively influential on people's self identity.

KOLs, especially those who are influential on the sexual minority field, contributed to the LGBTQ community by helping people to feel accepted, and providing solutions due to people's own circumstances. Sina Weibo, according to data collection " 15 Top Social Media Sites \& Platforms" conducted by James Everett Youngblood on SmartBlogger, updated in June 30, 2021, is the 9th popular social media platform among all other sites around the world[1]. Bloggers e.g., BanDao" ",Hoker, TongZhi Zhi Sheng", "internet-garcon-pdf" are examples of role models that have positive impact and made improvement on LGBTQ community. Each of these bloggers have more than nine hundred thousand followers, which made them undoubtedly users that have influential power. These bloggers would collect information of LGBTQ events around the world, and share these information on the platform. For example, on July 23, @同志之声 posted a blog that says "163 athletes that have already announced themselves to be LGBTQ will participate in the Tokyo Olympiad"[3][4]. Information like this would encourage LGBTQ followers to pursue their personal goals. People can also send messages to those bloggers, asked them questions about self identity related issue. Those bloggers would post those questions without saying the name of people who asked those questions, and others can answer those questions under the comments. In this way, people can find acceptance, and overcome their confusion.

Ordinary citizens are also passionate on improving the whole LGBTQ community using their limited but strong and faithful power. People who are not as organized and professional as the KOLs still use the spreading power of the internet to speak up for themselves or others about LGBTQ issue, whether to protest against unfair treatments, or just share their personal thoughts and anecdotes. In October 2020, in Spain, fifteen-year-old Mikel Gomez was expelled from school and had been send to the psychologist's for wearing skirt to school. This has prompted teachers across the country to join the "gender-neutral clothing" movement. Throughout this whole event, social media had played a major role. Gomez had posted a video on
Tik tok. In the video, he explained that he wore skirt to school in the purpose of challenging the gender norms for clothing, and to support female liberation; however, after doing so, he has been pulled out from class and brought to the psychologistss. After Gomez post this video, due to the spreading of internet, it became a heated topic across Spain. People started to protest. In November 4, hundreds of male students wore skirt to school. Many teachers and students in Spain joined this movement, supporting gender-neutral clothing. Throughout the whole event, Mikel Gomez, Jose Pinas, and Borja Velazquez are all role models that shared their personal stories of being discriminated for being LGBTQ, and encouraged others to join the movement on the internet [5]

Not only individuals are making progress on the sexual identity issues, so do many main stream media and news reports. Compared to individual bloggers, those organizations have more authority, and more accesses of accurate information and knowledge. For instance, on July 27, 2021, The Beijing News and Tecent News report conducted a news topic about the transgender community in China. Doctor from Beijing Third Hospital, who is the director of the transgender sequence medical field, talks about a case of a teenage transgender girl, and also explains knowledge about the transgender community to the audience [2]. Role models like those medias can spread information about the sexual minority groups, and help to extend the acceptance of these groups.

From the real-life examples provided above, the diversity and variety of the role models on the internet is conceivable. Therefore, the potential influential power of those role models are spectacular. Whether the role models are an organization, a group of people, or individuals, they all share the same goal: to speak up for the LGBTQ community, the minorities, to respect equality for and different values of each and every human-being.

\section{RELATIONSHIP BETWEEN NOWADAYS AND HISTORY}

LGBTQ is the trait which is decided by genes, people have their own sexual orientation when they were born.Some ancient countries cannot accept this kind of thoughts, old people also think it is a symptom for sick. Although a few people were conscious of they have difference with other people, they had no courage to express it. Just like our group's interview, most of interviewees knew their sexual orientation in their childhood,few of them realized they are LGBTQ people after searching the internet or the apps.

America is the first country which is encourage people to pursue their love include LGBTQ. In 2009, Grindr is an app to help male gay find their partner and know the geography position each other. The other app which named Blued was appeared in 2012 and influenced 
Chinese gay more, people can search other people's information such as age, photos, habits, sexual orientation and know the real position. It is an app which was popular all around the world. After 2013, more and more countries allowed people in freedom sexual orientation, even some countries can register marriage for LGBTQ.

With the developing of the internet, the number of LGBTQ population rises sharply during these years. From the government's statistic feature, LGBTQ people indicated an increasing tendency from 2014 to 2022. There were 350 million IGBTQ people in the world which occupied 4.8 percent in total. Form 2016 to 2022, the LGBTQ population inclined 0.3 percent in average. The professional researchers predict that LGBTQ will account for higher rate than before, this may be because the development of the social media or the internet, people can familiar with IGBTQ and realize themselves truly from many kinds of social software.

Our group interviewed 75 teenagers from online questionnaires, People who know about some superstars are LGBTQ occupy $88.89 \%$ in total, other people don't know it(interview). Most of people point that Cai Kangyong, Leslie Zhang, who are in China entertainment, other persons also know about foreign stars. The results indicate half of people influenced by superstars who are LGBTQ. Most of people are encouraged by them and pursue the true love, gain acceptance from other people, the most important point is that famous people can give them confidence about their love and braver to face the life. There are about 30 percent of people think IGBTQ superstars have no influences for them. The remain of interviewees, some of them think LGBTQ superstars can give them both positive and negative effects is tripled of people who think superstars only give them bad influences. In conclusion, LGBTQ + doesn't consider role models in social media affects themselves in sexuality, but helpful in gaining acceptance.For heterosexual people, role models in social media help them accept the existence of LGBTQ+ to a large extent.

We also interviewed non- LGBTQ people though the questionnaire, the result shows that the majority of the people who filled the questionnaire would hold positive attitude towards their friends if they are LGBTQ. On this basis, we could conclude that in general, over a half of the population would hold positive opinions upon LGBTQ community. Many interviewees know about LGBTQ from social media, such as Wechat, Instagram or films. Some of people have LGBTQ friends. Most of people accept LGBTQ and respect them, social media help people learn about this kind of knowledge. Someone also know themselves clearly and sexual orientation definitely through social media.

In Western ancient culture, people's status, gender, have the right to inherit or not had been decided when they were born. Fork considered that ancient rights controlled people's bodies and their death to prove themselves. In comparison, modern culture thinks that rights controlled all of things surrounded humans include their spirits and thoughts. Modern Western social entertainment, sex, health, exercise even education are all govern by the net of rights.From the introduction to identity' by Tao Jiajun indicates that desire is the lack between needs and require which cannot be covered. Desire is the product, and people are the machines to produce desire which is govern the humanity.

- Contemporarily, Western countries advocate people read classical books again to consolidate the status of male chauvinism, because it can benefit leaders' govern and ensure different kinds of criterion of identity. Some famous production also criticize the phenomenon such as 'sex policy' by Kate Millet.

- $\quad$ Old people remain the old conception about sexual orientation is decided by gender, they are not brave enough to accept it. Some of them think reproduce is the most important result for sex instead of pursuing true love. Different sexual orientation is a kind of disease by their conception. We are no longer to abide parents ' stand points to choose our lives, every country chase human right, but they also ban marriage between LGBTQ. The government disagree LGBTQ now, so that old people do not want to change their conception. In my opinion, social media is a good way to help people know and accept LGBTQ. Internet can also change the laws, the tendency of media agrees to pursue true love and encourage people to do anything they want to do. It is no doubt that LGBTQ acceptance is numerous and develop in a positive direction.

- In conclusion, people will accept LGBTQ more than before and governments will allow LGBTQ marriage in the early future, especially in western countries will have many teenagers aware their sexual orientation and do not afraid to be laughed by others.

\section{CONCLUSION}

It seems an increasing number of people concern that the influence to LGBTQ group which is a term for lesbian, gay, bisexual, transgender and queer or questioning from social media. However, whether the internet has positive effects to LGBTQ teenagers or not is a controversial issue. For this question, we had an investigation and argued that teenagers' self identity effected by cultures and the internet.

Virtually, with the growing of internet and social media, more and more teenagers aware their sexual 
orientation from software or hosts in famous websites. Few years ago, the app which named Grindr in order to help gays find their couple and know their geography position to make them missing each other. The other app which named Blued was appeared in 2012 and influenced Chinese gay more, people can search other people's information e.g., age, photos, habits, sexual orientation and know the real position. It is an app which was popular all around the world. The identity of this group is shown by the existence LGBTQ Pride Month, which is commemorated in the month of June per year.

From our questionnaire, we interviewed IGBTQ teenagers and non- LGBTQ teenagers. Most of LGBTQ teenagers said that they awared their sexual orientation when they were young instead of depending on the internet. Their sexual orientation depend on their genes, but the internet influenced their conceptions to LGBTQ or self identity.

From our investigation, we found that the growth of social media has positive and negative aspects to LGBTQ teenagers. The advantage is that the internet can help LGBTQ persons pursue their true love consistently and cause them do not afraid others' comments. The disadvantage is that some of teenagers are not LGBTQ but they think it is so popularity so that they blind to follow the 'fashion' which is not responsible to themselves.

In summary, the growing of social media can not help teenagers know about their sexual orientation definitely, but it can lead to LGBTQ teenagers consist their own identity. Teenagers' self identities influenced by people surrounding them who had been affected by social media. These results offer a guideline for future development of role models in social media helping more teenagers to carry out self-identity.

\section{REFERENCES}

[1] Smart Blogger. 2021. 15 Top Social Media Sites \& Platforms (A Handy 2021 Guide). [online] Available at: <https://smartblogger.com/socialmedia-

sites/\#: :text=15\%20Top\%20Social\%20Media\%2 0Sites $\% 20 \% 26 \% 20$ Platforms $\% 20 \% 28$ A, \%28both $\% 20$ are\%20owned\%20by\%20...\%20More\%20item s...\%20> [Accessed 27 August 2021].

[2] Weibo.com. 2021. Sina Visitor System. [online] Available at: <https://weibo.com/tv/show/1034:4663530981621 798?from=old_pc_videoshow $>$ [Accessed 27 August 2021].

[3] Outsports. 2021. At least 185 out LGBTQ athletes at Tokyo Olympics, a record by far. [online] Available

at: <https://www.outsports.com/olympics/2021/7/12/2 2565574/tokyo-summer-olympics-lgbtq-gayathletes-

list?continueFlag=ecb8fe3a4c900833ef3def0ca796c 4ce> [Accessed 27 August 2021].

[4] Weibo.com. 2021. Sina Visitor System. [online] Available at: <https://weibo.com/1664065962/Kq7E01GJU> [Accessed 27 August 2021].

[5] PinkNews - Gay news, reviews and comment from the world's most read lesbian, gay, bisexual, and trans news service. 2021. Defiant teachers wear skirts to school after student cruelly sent to psychologist. [online] Available at: <https://www.pinknews.co.uk/2021/06/07/spainteachers-wear-skirts-school-clothes-have-nogender-mikelgomez/?continueFlag=ecb8fe3a4c900833ef3def0ca 796c4ce> [Accessed 27 August 2021].

[6] Zhiwang - Queer considerations: Exploring the use of social media for research recruitment within LGBTQ communities [Online] Available at: https://schlr.cnki.net/zn/Detail/index/SJIXLAST/SJ IX9457B7344B1486731F450F736126C571

[7] Zhiwang - Effects of Issue Ownership, Perceived Fit, and Authenticity in Corporate Social Advocacy on Corporate Reputation [online] Available at: https://schlr.cnki.net/zn/Detail/index/SJESLAST/SJ ES1E36D90AF0A362E85BD575D80F2E4354

[8] Zhiwang - Restricted modes: Social media, content classification and LGBTQ sexual citizenship [online] Available at: https://schlr.cnki.net/zn/Detail/index/SJIXLAST/SJ IXBB49F028B7AE5D0A93FD4C4A785CAD5E

[9] Zhiwang - "Sometimes You Get Married on Facebook": The Use of Social Media among Nonmetropolitan Sexual and Gender Minority Youth. [online] Available at: https://schlr.cnki.net/zn/Detail/index/SJPDLAST/SJ PDFD5CDD5F5AB37E18EBB01BC507D96ACB

[10] Zhiwang - Use of social media to assess the impact of equitable state policies on LGBTQ patient experiences: An exploratory study [online] Available at: https://schlr.cnki.net/zn/Detail/index/SJESLAST/SJ ES95B727FDC185A784B2E574236961034E 